\section{Is vasculogenesis crucial for the regrowth of irradiated tumours?}

\author{
Sergey V. Kozin, Dan G. Duda, Lance L. Munn and Rakesh K. Jain
}

We have read with interest the Review by Begg, Stewart and Vens (Strategies to improve radiotherapy with targeted drugs. Nature Rev. Cancer 11, 239-253 (2011)) $)^{1}$, which included discussion on the potential benefits of modulating the tumour microenvironment to improve radiotherapy. In their Review, the authors state that, "vasculogenesis (vascular formation from circulating BMDCs (bone marrow-derived cells)) has been shown to be crucial for the growth of tumours that recur after radiotherapy". We agree that new vessel formation is an important determinant of tumour regrowth after irradiation. However, we question the authors' conclusion that vasculogenesis is crucial in this context.

To our knowledge, there are no published reports showing greater recruitment and incorporation of non-irradiated endothelial progenitor (or precursor) cells (EPCs) or any other BMDCs - into the vessels of tumours that are regrowing after local irradiation compared with their ionizing radiationnaive counterparts. In fact, three animal studies have directly tested this hypothesis either in irradiated tumours or using tumours implanted in pre-irradiated beds - situations in which the local endothelial cells that are capable of neovascularization have been substantially damaged by ionizing radiation. None of these studies found evidence for increased BMDC incorporation into the vessels of such tumours ${ }^{2-4}$. Moreover, one of these papers - overlooked by Begg and colleagues in their Review - emphasized that the recruitment of EPCs does not facilitate tumour regrowth after local irradiation ${ }^{4}$.

The discrepancy seems to be due to the initial, imprecise use of the term 'vasculogenesis' elsewhere ${ }^{2,3}$. Although studies have shown that BMDCs increasingly infiltrate into tumours after irradiation and facilitate relapse $\mathrm{e}^{3-5}$, they do not incorporate into the vasculature. Thus, these BMDCs do not participate in vasculogenesis as defined by Begg and colleagues and in the literature in general ${ }^{1,6}$. Indeed, the BMDCs that are recruited by irradiated tumours are generally of myeloid lineage - including 'alternatively' activated macrophages and tyrosine protein kinase receptor (TIE2)-expressing monocytes (TEMs) - that facilitate postirradiation tumour revascularization through paracrine mechanisms ${ }^{2-5}$. Distorting the definition of vasculogenesis not only confuses the literature but also misrepresents the biology - in this case by implying that the cells are doing something that they are not.

Interestingly, several recent discoveries might add new twists to the issue. It has been shown that a fraction of endothelial cells in growing tumours may be derived, through trans-differentiation, from cancer stem-like cells $^{7-9}$, which may be more radioresistant than other neoplastic cells ${ }^{10,11}$. Alternatively, EPCs that are not of bone marrow origin might potentially be recruited to tumours from nonirradiated adjacent tissues and/or the circula$\operatorname{tion}^{12,13}$. It is conceivable that all of these cells may be participating in vasculogenesis in tumours that recur after irradiation. But at this stage, this remains a hypothesis. Only when these or other proposed mechanisms of EPC differentiation and incorporation are proven in irradiated tumours should we conclude that vasculogenesis is crucial in relapsing tumours.

Sergey V. Kozin, Dan G. Duda, Lance L. Munn and Rakesh K. Jain are at the Steele Laboratory for Tumor Biology, Department of Radiation Oncology,

Massachusetts General Hospital, Harvard Medical School, Boston, Massachusetts 20114, USA. Correspondence to R.K.J. e-mail: jain@steele.mgh.harvard.edu doi:10.1038/nrc3007-c1

1. Begg, A. C., Stewart, F. A. \& Vens, C. Strategies to improve radiotherapy with targeted drugs. Nature Rev. Cancer 11, 239-253 (2011).

2. Ahn, G. O. \& Brown, J. M. Matrix metalloproteinase- 9 is required for tumor vasculogenesis but not for angiogenesis: role of bone marrow-derived myelomonocytic cells. Cancer Cell 13, 193-205 (2008).

3. Kioi, M. et al. Inhibition of vasculogenesis, but not angiogenesis, prevents the recurrence of glioblastoma after irradiation in mice. J. Clin. Invest. 120, 694-705 (2010).

4. Kozin, S. V. et al. Recruitment of myeloid but not endothelial precursor cells facilitates tumor re-growth after local irradiation. Cancer Res. 70, 5679-5685 (2010).

5. Li, F. et al. Regulation of HIF-1 alpha stability through S-nitrosylation. Mol. Cell 26, 63-74 (2007).

6. Carmeliet, P. \& Jain, R. K. Molecular mechanisms and clinical applications of angiogenesis. Nature 473, 298-307 (2011).

7. Ricci-Vitiani, L. et al. Tumour vascularization via endothelial differentiation of glioblastoma stem-like cells. Nature 468, 824-828 (2010).

8. Soda, Y. et al. Transdifferentiation of glioblastoma cells into vascular endothelial cells. Proc. Natl Acad. Sci. USA 108, 4274-4280 (2011).

9. Wang, R. et al. Glioblastoma stem-like cells give rise to tumour endothelium. Nature 468, 829-833 (2010).

10. Bao, S. et al. Glioma stem cells promote radioresistance by preferential activation of the DNA damage response. Nature 444, 756-760 (2006)

11. Baumann, M., Krause, M. \& Hill, R. Exploring the role of cancer stem cells in radioresistance. Nature Rev. Cancer 8, 545-554 (2008).

12. Aicher, A. et al. Nonbone marrow-derived circulating progenitor cells contribute to postnatal neovascularization following tissue ischemia. Circ. Res. 100, 581-589 (2007).

13. Ergun, S., Hohn, H. P., Kilic, N., Singer, B. B. \& Tilki, D. Endothelial and hematopoietic progenitor cells (EPCS and HPCs): hand in hand fate determining partners for cancer cells. Stem Cell Rev. 4, 169-177 (2008).

Competing interests statement

The authors declare no competing financial interests. 